\title{
Strategies and considerations in shaping Cyber-Physical Systems education
}

\author{
Martin Törngren and Martin Edin Grimheden \\ Mechatronics/Machine Design \\ KTH, 10044 Stockholm, Sweden \\ +4687907797 \\ (contact: \{martint,mjg\}@kth.se)
}

\author{
Jonas Gustafsson and Wolfgang Birk \\ Computer Science, Electrical and Space Engineering \\ Luleå University of Technology, 97187 Luleå, Sweden \\ +46920491965 \\ (contact: \{gusjon,wolfgang\}@ltu.se)
}

\begin{abstract}
Embedded and Cyber-Physical Systems education faces several challenges as well as opportunities as every-"thing" becomes connected, and as technology development accelerates. Initiatives such as CDIO, as well as several other academic and industry initiatives to create new CPS programs illustrate strong interests and awareness of these challenges. We provide an overview of foreseen educational needs, existing state of the art in education and an analysis of the subject of CPS with the purpose of understanding the implications for education. The investigation points to key issues in curriculum design regarding balancing depth and breadth, theory and practices, academic and industrial needs, and core technical skills with complementary skills. Curricula in CPS could, if the right balance is achieved, educate CPS engineers of the future that are "ready to engineer". We conclude by synthesizing high level guidelines in terms of strategies and considerations for CPS curriculum development.
\end{abstract}

\section{Categories and Subject Descriptors}

K.3.2 [Computers and Education]: Computer and Information Science Education - accreditation, computer science education, curriculum, information systems education

\section{General Terms}

Management, Performance, Human Factors

\section{Keywords}

Curriculum guidelines, Cyber-physical systems, Embedded systems, Engineering education

\section{INTRODUCTION and MOTIVATION}

What skills and capabilities are required by the engineers of tomorrow? Furthermore, how should the corresponding educational programs be formed in order to provide experts ready to engineer the Cyber-Physical Systems that will greatly impact our future society?

\footnotetext{
This work is based on an earlier work: Education and training challenges in the era of Cyber-Physical Systems: beyond traditional engineering. In Workshop on Embedded and CyberPhysical Systems Education, WESE, (C) ACM, 2015. http://dx.doi.org/10.1145/2829957.2829965
}

Copyright of this extended version retained by the authors.
Embedded and Cyber-Physical Systems education is facing several challenges as every-"thing" becomes connected, and as technology development accelerates. Distinguishing "embedded" from "IT" used to be simple. With the increased levels of networks and connections between different systems - and the use of software-intensive systems in all sorts of new applications including mass-market products - our traditional separation into various disciplines and domains is no longer adequate. Moreover, system complexity increases, requiring process and organizational change. This provides an increased need for engineers with system knowledge, which goes beyond the traditional disciplines.

This development raises the question whether our current educational programs are sufficient. Do we need to develop new "multidisciplinary" programs, or should we aim for a new CPS discipline and corresponding programs? How long should engineering educations be? Should more emphasis be placed on lifelong learning - for example in terms of continued training for practicing engineers?

The main contributions of this paper are threefold:

1. We provide an overview of foreseen educational needs and existing state of the art in education.

2. We provide an analysis of the subject of CPS, with the purpose of understanding the implications for education.

3. Based on this analysis, we propose guidelines to assist in CPS curriculum development or updates.

Specific attention is devoted to the multidisciplinary nature of CPS education, with its connection to multiple academic disciplines and (existing as well as new) application domains.

To obtain information regarding the educational aspects of CyberPhysical System, a number of workshops have been conducted. Participants at the workshops span from Academia to Industry. To get a representative view of how the term "Cyber-Physical Systems" is perceived and educational aspects are anticipated in different parts of the world, workshops have been conducted in the following regions, Asia (India), America (USA), Pacific (Australia), and Europe (Sweden).

The article is laid out as follows. Section 2 elaborates CPS characteristics and trends, and discusses educational needs mainly from an industrial point of view. Section 3 describes state of the art in terms of existing educational practices and curriculum recommendations. Based on Sections 2 and 3, Section 4 identifies and discusses challenges and gaps, whereas Section 5 describes the synthesized recommendations. Section 6 concludes the paper with recommendations for further work. 


\section{CPS CHARACTERIZATION AND SOCIETAL EDUCATIONAL NEEDS}

CPS is a relatively new term originating from the US in 2006, with the realization that the interaction between the intelligence provided by interconnected processing systems and the physical world could not be ignored or considered of secondary importance. A corresponding early definition is as follows: CPS use computations and communication deeply embedded in and interacting with physical processes to add new capabilities to physical systems. These CPS range from minuscule (pace makers) to large-scale (the national power-grid), [20].

Multiple interpretations and definitions of CPS have since been presented. Industrial representatives rightfully point out that CPS, according to the above definition, are indeed not new but already existing and manifested by for example existing industrial distributed control systems. The increasing system connectivity and capabilities of computational systems however drive the creation of entirely new systems, characterized by:

- Deployment of CPS in mass-products for use in all kinds of applications in society, for example exemplified by smart-phone enabled services.

- Opportunities for and introduction of new cross-domain applications, exemplified by intelligent transportation systems which integrate among other communications, mobility, entertainment and safety-related services. New cross-domain collaboration typically call for new business models and interoperability standards.

- Increasing openness, adaptability and autonomy.

These characteristics reflect the evolving scope of CPS, from traditionally closed systems, with single jurisdiction, limited adaptability and autonomy, [21].

The increased level of integration between the domains of the physical world, embedded systems and the internet/IT world, raises questions about needs for future engineers, both in terms of their numbers and desired skills/knowledge - these two topics are examined in the following.

\subsection{Future of CPS job market}

According to a recent investigation by ARTEMIS and ITEA2, the global market of Digital Technology is estimated at USD 3,300 billion, corresponding to approximately 50 million jobs, for which the Europe share translates to about 9.1 million jobs (about 20\%), [6]. The term Digital Technology was introduced (due to a lack of coherent terminology) to account for software, embedded software, IT services, internal IT as well as hardware encompassing semiconductors, PCs, tablets, servers, storage, and peripherals.

The global market for Digital Technology is predicted to have a continued strong growth with expectations for a strong increase in demand of embedded and CPS engineers, [6]. The same investigation also highlights that the key selling product features are increasingly dominated by CPS and embedded systems as they become part of more and more products and systems.

Consequently we foresee an increasing need for CPS engineers and also for CPS knowledgeable people in general given the increasing societal reliance on such systems.

Our experiences from nearly 30 years of education in Mechatronics and Embedded system, confirm the importance of CPS and embedded systems education, and the industrial demand, for example resulting in a relatively recently formed Master program in Embedded systems at KTH. The creation of the program was driven by industrial partners of KTH centre for embedded systems (ICES), [22].

Regions increasingly depend on strong universities and education as one important attractor for CPS industry. Closer industry and academia regional collaboration is becoming more and more important.

Similar findings are reporting in [27] and [28] where also the need to deal with the structural constraints, following the increasing disciplinary specialization and university organization, are emphasized.

\subsection{Required skills of CPS engineers}

Development, production, operation and maintenance of CPS require an increasingly large skill-set, beyond the capabilities of (most) single individuals.

The corresponding knowledge encompasses physics (and analog technology), geometry, and digital/software technology, providing heterogeneity in concepts, time and space. A key challenge in CPS engineering is to be able to integrate such heterogeneous and complementary perspectives to form a coherent system. This requires that models and methods from a number of disciplines, such as computer science, electronics, control and mechanics are consistently combined, [23].

While knowledge has increased in all areas of technology, specific knowledge is also required for developing "systems" and integrating heterogeneous technologies. In a CPS, dependencies across components and aspects will require consideration of "nonlocal" effects. Closely related to this, a modern engineer will have to interact with many other engineers and stakeholders, thus requiring also communication and project management skills going well beyond technology.

Requirements for a sustainable society further require consideration of a number of life-cycle concerns including environmental effects, recycling and disposal.

Because of the important influence of CPS in society, and their interactions with various stakeholders, the skill-set moreover many times extends to economics, human machine interaction, legislation and social sciences.

\subsection{Importance of teaching and continued training}

Excellence in education and a skilled work force will be of paramount importance for grasping CPS opportunities. During our investigations of CPS education and interactions with teaching institutions, we have identified the following important aspects:

- Teaching has a low status in universities in Europe, and also in high schools and proceeding schools. Initiatives to improve the status of teaching and management of teaching are urgently needed; this is particularly valid for CPS but it is our understanding that this topic is part of a larger concern valid not only for CPS.

- Special efforts are needed to promote CPS training for practicing engineers. For example, engineers and managers in a traditional mechanical engineering industry may not be well trained in cyber/embedded parts of CPS. Likewise, embedded systems engineers may not be skilled in Internet technologies, and vice versa. 


\section{TOWARDS A STATE OF THE ART IN CPS EDUCATION}

We aim to characterize knowledge and research related to CPS education into two distinct domains; (i) current research and activities related to engineering education, and (ii) discipline specific educational efforts.

The former encompasses results from initiatives such as CDIO [7], [8], [9], criteria from ABET [10] and educational research findings related to the scope of this article. In the latter we include discipline specific efforts related to embedded systems, CPS, systems engineering and mechatronics.

We further provide examples of representative CPS educational programs and trends. To set the stage, we start with a discussion about the characterization of the subject of CPS.

\subsection{Related engineering education research and activities}

\subsubsection{The CDIO initiative}

The CDIO initiative is a framework for educational program development. The initiative stems from MIT around 1997, with an international spread around 2000. The initiative was launched with the idea of reforming higher engineering education both towards engineering fundamentals, and, at the same time, to create engineers with an ability to engineer. Companies and organizations such as Boeing and ABET presented lists of abilities they wanted students to possess [7]. These abilities relate to real-world engineering situations and were a reaction to many universities transforming education more towards engineering science.

The CDIO initiative states that graduating engineers should be able to: "conceive, design, implement and operate complex valueadded engineering systems in a modern team-based environment and are mature and thoughtful individuals", [7].

So far, more than 100 universities have joined the CDIO initiative, demonstrating that the notion of "creating engineers ready to engineer" is spreading fast.

\subsubsection{ABET criteria for Engineering Education}

The Accreditation Board for Engineering and Technology (ABET) has established criteria for accrediting engineering programs. Some of these criteria are based on student outcomes. An educational institution can seek accreditation with ABET, and in some countries it is of utmost importance for educational programs to be ABET qualified. The criteria relating to student outcomes are grouped into eleven outcomes. When designing a CPS curriculum, it is certainly not a disadvantage to glimpse at these criteria for assessing outcome of engineering programs. The first five criteria are presented below:

- an ability to apply knowledge of mathematics, science, and engineering

- $\quad$ an ability to design and conduct experiments, as well as to analyze and interpret data

- an ability to design a system, component, or process to meet desired needs within realistic constraints such as economic, environmental, social, political, ethical, health and safety, manufacturability, and sustainability

- $\quad$ an ability to function on multi-disciplinary teams
- $\quad$ an ability to identify, formulate, and solve engineering problems

The remaining six criteria deal with professional and ethical responsibility, communication, engineering contexts, life-long learning, contemporary issues and the ability to use modern tools.

ABET recommends that engineering programs include a capstone course in the curriculum. A capstone course aims to bring together major aspects of the academic disciplines relating to the subject of the program, to train students to utilize knowledge and skill from previous courses. A capstone course can train professional skills and further train students to be "ready to engineer", borrowing the phrase from CDIO.

While these criteria are for engineering programs, and not directly applicable to programs in, for example, physics, ABET does include computer science programs.

\subsubsection{Balance between Knowledge and Skills, Theory and Practice}

The birth of PBL, Problem Based Learning, came as a reaction to the then too theoretical medical training programs that existed. Medical programs were thought to teach the science of medicine but not to prepare future practitioners very well. Barrows et al. reformed a medical school program in Ontario, Canada, in the late 1960 s by introducing PBL, [11].

Simultaneously, a deeper understanding on how people learn started to be more common and new pedagogies evolved. Among the research that popped up in this wake were the very much quoted studies on deep- and surface approaches to learning undertaken by Marton and Säljö, [12]. In these studies, Marton and Säljö showed that students develop, or take, different approaches to learning. While in some cases, a surface approach can be very efficient to memorize large quantities of material, a deep approach might result in an understanding of the underlying concept and meaning, while the details for example might not be remembered. These studies do not show that one approach is better than the other. The studies show that students take different approaches, and that the different approaches train differently. A subject, or profession, might require both knowledge and skill, both theory and practice, and it is important to keep a balance that is relevant for the subject and future profession.

\subsubsection{Summarizing related research and activities}

The results and experiences from CDIO show that a shift of focus in engineering programs has been (is being) made, with more focus on professional, applicable engineering skills but without losing engineering fundamentals or scientific rigorousness. CDIO shows that this reform can be made, and that it leads to both stronger fundamentals and a "capability to engineer" [9].

The ABET criteria show that US universities are assessed according to students abilities to apply knowledge, both of the subject of the program, but also of mathematics and science in general. These criteria also show that an engineering program in general should result in ability to conduct and perform experiments, analyze data, design systems, components and processes, function in multidisciplinary teams, solve engineering problems etc.

The history of PBL and more current research on learning approaches and strategies point toward the importance of finding the balances between theory and practice, between knowledge and 
skills, and to develop programs, courses and modules appropriately.

\subsection{Education research in selected technical fields}

In this section, we present studies on subjects similar, and relevant to CPS, with a focus on research on curriculum design for CPS. An increased interest in the area can be noted in several academic communities, for example through the WESE workshop on embedded systems education at the Embedded Systems week (that in 2012 changed name to include CPS in the title), as well as with the first CPS education workshop at CPS week, [24].

Previous studies by Törngren and Grimheden were carried out on the subjects of Mechatronics, [13] and Embedded Systems, [14] to create a foundation for further curriculum design. In these studies, both subjects are classified as thematic (as opposed to disciplinary) and functional (as opposed to formal).

The thematic identity implies that the subject is hard to define, and that it is rather pointless, or at least very difficult, to try to find a universal consensus on the definition, content and teaching strategy, as opposed to disciplinary subjects such as mathematics where university courses seldom vary between universities or decades.

With CPS, most universities approach the subject differently, depending on university organization, student popularity, market interest and individual interest among professors. All in all, the subject typically emerges as a theme, an idea, from an interaction, collaboration or discussion between domains, university professors or departments, for example, computer science, mechatronics, embedded systems, automatic control etc. The theme(s) that evolve from this combination, meaning the research ideas and proposals, the courses, student projects etc., typically define the subject. Examples of typical themes, or strands, that we have noted are:

- Mathematical approach - CPS needs to reconcile the underlying (disciplinary) theories and find common shared, abstractions, for example among computer science and control theory.

- $\quad$ Extension approach - where a particular discipline and curriculum, e.g. in computer science, is extended with one or two courses to provide complementary competence (e.g. on the "physical" aspects of CPS).

- Project and problem oriented approach - Theory and practice needs to be bridged, and systems engineering perspectives (requirements, architecture, and integration) provided.

The legitimacy of the subject, is characterized as more functional than formal, [13]. Hiring companies requests functional skills more than formal knowledge. The hiring agent will most likely ask the candidate for his or her ability to apply knowledge, to design systems and products than to give a list of which books and subjects the candidate has read. The formal knowledge is essential, but the value lies in the ability to apply the knowledge.

The above is valid if the intention is to create a program that teaches students to design, implement, use and operate cyberphysical systems, a CPS engineering program.

\subsubsection{The concept of synergy}

As with Mechatronics and Embedded Systems, most of the more commonly used definitions of the subject are based on the concept of synergy. An example of this is the definition currently used by the US National Science Foundation, NSF:

Cyber-physical systems (CPS) are engineered systems that are built from and depend upon the synergy of computational and physical components. Emerging CPS will be coordinated, distributed, and connected, and must be robust and responsive.

As presented in previous studies, synergy is hard to teach, [14]. "CPS are engineering systems that are built from and depend upon the synergy...". Synergy can be studied, but to master synergy the underlying subjects need to be understood. This has to be practiced, and the challenge is to be able to create this synergy without being expert in (all of) the underlying subjects. Therefore, if the subject of CPS is defined based on the concept of synergy, it can be argued that the expertise of the CPS engineer lies in the ability to understand and utilize this synergy - to differentiate from experts in the fields of computer science, control, communication etc.

To reach this specific expertise, there is a need to find a suitable balance in a system- and disciplinary perspective. In composing CPS engineering teams there is a need to gather persons providing the required disciplinary expertise (in some form) and with person(s) that have the ability to provide a systems- and integration perspective, which of course could be the CPS engineer.

\subsubsection{The whole is greater than the sum of its parts}

A CPS graduate cannot have studied as much computer science as a CS graduate, not as much electrical engineering as an EE graduate etc. The CPS graduate must possess other competences and skills, skills such as the ability to create synergy between these subjects.

PBL and medical programs were mentioned previously. A medical student might work as a physician 50 years after graduation. The medical field hardly stays undeveloped for that long, there will be a lot of new things to learn. The program must take that into account and teach generic skills and the ability to adapt. In a related comparison, the field of medicine evolves quite fast with new discoveries, new methods and tools - but the length of the program stays roughly the same. New things cannot simply be added into the curriculum, the curriculum must constantly be revised and learning goals replaced.

Similarly, an engineer needs to be kept up to date with new knowledge, skills, and trends. The concept of life-long-learning is applicable in this context but need to be more actively promoted through professional training at post-graduate level.

\subsubsection{Systems engineering}

Systems engineering is an interdisciplinary field that overlaps control, industrial engineering, project management etc. Systems engineering also emerged from an industrial need and there was a rather clear idea of what competencies and skills that was required of a systems engineer. The subject has matured enough so that programs, courses and modules in systems engineering exist at many universities, meaning the programs are necessarily not made of existing courses in the underlying subjects but rather of courses in systems engineering. The field includes a lot of "new" topics such as systems modeling languages. 
INCOSE, the International Council of Systems Engineering, presents a list of 165 programs in systems engineering in the US, divided into systems-centric and domain-centric, [15]. The systems-centric programs treat systems engineering as a separate discipline while the domain-centric programs starts in a separate domain such as mechanical engineering, electrical engineering, and teaches systems engineering as a specialization of that subject.

INCOSE does not recommend curriculum ideas or suggestions, it merely "advocates that academic institutions offer more engineering degree programs with strong components in Systems Engineering, and supports academic institutions that have chosen to offer programs that lead to degrees in Systems Engineering." [15]. It can be noted that the recommendations suggest the inclusion of a capstone course, as mentioned in section 3.1.2.

\subsubsection{Embedded systems and mechatronics}

The subjects of embedded systems and mechatronics share similarities with systems engineering in the sense that they are multi-disciplinary subjects, academic subjects that have matured and are established at many universities. An embedded systems or mechatronics program can likewise be based on either a separate domain such as electrical engineering, or be taught as a separate domain.

For both subjects, suggestions for curriculum design exist. The ARTIST Education group published guidelines "for a graduate curriculum on embedded software and systems", [16]. These guidelines acknowledge the challenge of multiple disciplines, of requiring expert skills and competences from several domains. These guidelines assume that a student has a BSc-level degree in computer science and base an MSc program on that assumption, designing an embedded systems specialization program, and proposing content for that program.

Studies on curriculum design for Mechatronics, for example as proposed by the EUMECHA-PRO, [17], instead promote a diversity of BSc-level degrees and topics in the MSc-level programs, emphasizing the special characteristics at the various universities, related to the various definitions and characterizations of the various academic constellations. In this proposal, mechatronics programs shifted focus between universities from for example precision motion control to robotics to complex product development.

\subsection{Example of CPS educational profiles}

To date, we are only aware of a few educational programs in Cyber-Physical Systems. In 2013, at the First workshop on CyberPhysical Systems Education (CPS-Ed 2013), a number of courses and modules were presented, [24]. One attempt at proposing a program was presented by Peter Marwedel et al. [18]. This program is divided into undergraduate and graduate level. For the undergraduate level, Marwedel et al. propose five parts, or topics; computer science, physics, electrical engineering, mathematics and CPS, meaning that the curriculum is divided between these five topics. Beside these topics, the authors propose a sixth topic that is voluntary and could be related to specific characteristics of the respective universities. For the graduate level, the study proposes to include more theoretical aspects, more depth and supplementary material.

The UC Berkeley EECS program strongly emphasizes CPS. At the core of the approach lies the teaching of core system modeling and model integration techniques, enabling model based CPS design, [25].

On the contrary, universities in Australia are more hesitant to introduce CPS programs due to the uncertain market opportunities for the engineers graduated in such programs and the relatively new scientific foundation for a CPS program. The approach taken by for example University of Melbourne and Monash University is to develop CPS specialization within existing mechatronics or electrical engineering programs. In that way, the foundation of the programs is well defined and recognized up to the BSc level.

Honoring the cross-disciplinary character of CPS education is then achieved by electable components in the MSc level program parts. There is a consensus from program directors, that CPS branded engineers need to be able to fall back to a solid and wellestablished competence for the Australian market. As soon as there is a sufficient market-pull for CPS branded engineers and CPS has established itself as an engineering discipline with sufficient scientific foundation, then this requirement on the education program can loosened.

Both University of Melbourne and Monash University pointed out system robustness and the ability to design systems to withstand errors "by design" as important knowledge area of CPSengineers. Also the ability to understand the challenges of other engineering disciplines was something that was raised several times.

EIT ICT Labs, the Knowledge and Innovation Community set up by the European Institute of Innovation and Technology runs a Master School with seven technical majors. Students of this school are invited to participate in "summer schools". In 2014, one of these summer schools was focused on Cyber-Physical Systems. The summer school ran for two weeks and mixed CPS lectures with team projects, use cases and lectures in business and innovation. The setup of the summer school was as follows:

Week 1: introduction of challenges on the themes of embedded systems, (networked) control systems, wireless sensor networks, and computer science. Lectures followed by specific tutorials and group work in order to let students connect theory with practice.

Week 2: focus on acquiring skills for preparing a business plan and the corresponding pitch presentations on one or combination of the introduced challenges.

\subsection{Education trends}

In this section we discuss how current trends in engineering education could influence development of a curriculum in CPS.

MOOCs, for Massive Open Online Courses, began to emerge in 2012 as a new development in distance education. A course or program in CPS could benefit greatly from being open and online, since universities could pool resources and the online setting could enable multi-disciplinary teamwork.

Example of a CPS-like MOOC is provided by Egerstedt [19] who enrolls 40.000 students in a robotics control course given by Electrical and Computer Engineering at Georgia Institute of Technology.

Another example is presented by Jensen et al [4], "Virtualizing Cyber-Physical Systems: Bringing CPS to Online Education". In this paper, the authors present a course given at UC Berkeley that has been transformed into a MOOC. Students are developing software for a robotic vacuum cleaner using LabVIEW Robotics Environment Simulator. Originally, in the non-MOOC-course, the 
students controlled a real robot, but with the MOOC setting, the authors argue that it's impossible to keep experimenting with hardware equipment if real scalability is wanted. Instead, the students use a simulation of the robot to verify their results.

\section{DISCUSSION}

A subject that crosses so many domains as CPS and at the same time requires a very strong theoretical base is difficult to define, grasp, master and teach. The metaphor of "T-shaped people" [26] can be used to describe the abilities required of a CPS engineer or graduate; the competence needs to consist of two types of abilities. The vertical bar on the " $T$ " represents depth and expertise in a single field and the horizontal bar represents abilities to work and collaborate with other "Ts", with people with other expertise. In this perspective, it's not crucial that the depth and expertise is in the exact right subject, the combination of depths within a team, and the ability of this team to create synergies between these depths is more important. This can be summarized into balancing the depth and the breadth, balancing core technical skills with complementary skills.

The examples of CDIO and ABET illustrate the balance between theory and practice, between knowledge and skills. In both cases, it can be argued that the balancing is the key success factor: practice needs theory, theory needs practice, skills need knowledge and vice versa. The CDIO case showed that an increased focus on practice leads to increase in engineering fundamentals, and theory.

\section{SYNTHESIS OF RECOMMENDATIONS}

The world is facing a technical paradigm shift (regardless whether we use the term CPS, IoT, or Industrie4.0). This shift implies that the amount of knowledge and skills required for product and service engineering is increasing along with the needs to continuously update your knowledge due to the evolving technology and society. Engineers are already working in project based (and often international) teams, implying that communication and collaboration skills are increasingly important, and that cross-discipline and contextual understanding also becomes increasingly important.

Excellence in education and a skilled work force will be of paramount importance for grasping CPS opportunities.

Given the previous analysis and combined with the outcome from a series of workshops with educators from the US, Europe, Asia, and Australia, we deduce the following strategies for developing or refining CPS programs.

In general, efforts should be stimulated and initiated to revise and improve programs, courses and training, considering the balancing required in educating CPS engineers, e.g. depth vs. breadth, theory vs. practice, and "profile positioning" of T shaped engineers. In doing so, the recruitment and academic context needs to be taken in account since it will influence the positioning of the chosen T-shapes. A complicating factor in CPS is the large variety of application domains.

Engineering programs need to provide engineers that are

(i) ready to engineer,

(ii) provided with long lasting knowledge and with new knowledge (for industry),

(iii) the ability to learn, and, (iv) able to communicate with, and understand the challenges of engineering fields beyond their own area of expertise.

Based on outcome from the workshops the authors come to the conclusion that a CPS-education beneficially can be founded on established engineering programs in related fields, such as electrical-, mechatronics-, mechanical, physics- or computerengineering. A specialization towards CPS, or complementing master degree in CPS would give the students the ability to broaden their perspectives in one or more areas within CPS. The CPS-part of the education will be found as the horizontal part of the "T"-model, where the vertical part is based on the established engineering education.

With the evolution of CPS as a theme and along with the development of science, we also see the longer term need to investigate dedicated CPS engineering education that encompasses and integrates the cyber and physical parts from day 1. While specializations will be necessary due to the broad scope of CPS, time will make us better understand topics that may be at the very core of CPS.

Universities should be stimulated and encouraged to take up best practices such as CDIO to reach these goals, see e.g. [9].

Incentives should be provided to stimulate academia and industrial collaboration in education, combining best practices and thereby addressing "non-academic", but nevertheless important, skills in industry (examples include configuration management and software build environments). Resultantly, professional training needs to become a shared responsibility between academia and industry and thereby enabling life-longlearning. Continued training may require specific consideration for educating practicing engineers in concepts that are new them; for example in teaching IT and embedded systems technology to mechanical engineers, or vice versa, in teaching physical system aspects to IT engineers.

To support (life-long) education and innovation there are also needs to develop and promote educational platforms, open labs, and maker-spaces.

A key aspect is that of strengthening the role of teaching and teaching leadership. The current academic system does not to a sufficient degree, provide incentives for teaching. More resources are also required for teaching environments including CPS labs.

From the workshops it was also possible to extract information knowledge and skill domains, which are of importance for CPS engineers. The following have been identified as most important:

- Error propagation in systems and failure management;

- Cross disciplinary communication;

- Distributed systems and scalability aspects;

- $\quad$ Process modeling and simulation, and co-design;

- Human-in-the-loop.

Again, the trade-off between depth and breadth in the education need to be considered here. In the design of the education these aspects can be considered by selecting depth topics as a combination of topics that are needed in relation to existing education programs whilst broadening is considered from the aspects of interoperability between engineering fields. 


\section{CONCLUSIONS AND FUTURE WORK}

In this paper we provided an initial analysis of foreseen educational needs and existing state of the art in CPS education.

In general, it can be stated that the CPS education programs are in development around the globe and that most institute take a rather pragmatic approach, where existing education programs are modified to fit the purpose. While this is a conservative comprise in relation to the achievable results, it means that graduates from such programs have the necessary skill set for accepted and accredited engineering fields.

Nevertheless, it is important to achieve a harmonization between the different programs and alignment with the emerging research field of cyber-physical systems.

We also identified issues that require further investigation.

- What are limitations in existing education, and how can such limitations be avoided in newly developed CPS programs?

- How should the long-term development of the subject CPS be pursued to improve quality of education and research?

It is the firm belief of the authors that further research on the topics of CPS itself including its technological foundations is required.

\section{ACKNOWLEDGMENTS}

This work has been supported in part by EIT ICT Labs.

\section{REFERENCES}

[1] Paul Caspi, et a., Guidelines for a graduate curriculum on embedded software and systems. ACM Transactions on Embedded Computing Systems (TECS). Volume 4, Issue 3 (August 2005), pages: $587-611$.

[2] Pyster, A., D.H. Olwell, T.L.J. Ferris, N. Hutchison, S. Enck, J. Anthony, D. Henry, and A. Squires (eds.). 2012. Graduate Reference Curriculum for Systems Engineering (GRCSE®). Hoboken, NJ, USA: The Trustees of the Stevens Institute of Technology. Available at: http://www.bkcase.org/grcse-2/ (accessed Aug. 2014).

[3] First workshop on CPS education, April $8^{\text {th }}, 2013$, Philadelphia, PA (part of CPSWeek 2013): http://cpsvo.org/group/edu/workshop - Accessed July 2014.

[4] Jeff Jensen et al. Virtualizing Cyber-Physical Systems: Bringing CPS to Online Education. CPSed 2013. http://www.eecs.berkeley.edu/ sseshia/pubdir/cpsed13mooc.pdf (accessed July 2014).

[5] Törngren M., Grimheden M., Adamsson N. Experiences from large embedded systems development projects in education, involving industry and research. SIGBED Review. $A C M$. ISSN:1551-3688. 12th issue, Vol. 4, No. 1 (Jan. 2007. Special Issue on the Second Workshop on Embedded System Education.

[6] ITEA ARTEMIS-IA High-Level Vision 2030: Opportunities for Europe, Autumn 2013. URL: https://itea3.org/publication/download/publication/961/file/I TEA_ARTEMIS_IA_high_level_vision_2030_v2013.pdf (accessed August 2014)

[7] www.cdio.org (accessed Aug 2014)
[8] Edward F. Crawley (2002). "Creating the CDIO Syllabus, A Universal Template for engineering education". Frontiers in Education, 2002. FIE 2002. 32nd Annual. Frontiers in Education 2. IEEE. doi:10.1109/FIE.2002.1158202. ISBN 07803-7444-4.

[9] Edward Crawley; Johan Malmqvist; Sören Östlund; Doris Brodeur (2007). Rethinking Engineering Education, The CDIO Approach. Springer. ISBN 978-0-387-38287-6

[10] http://www.abet.org/eac-criteria-2014-2015/ (accessed Aug 2014)

[11] Neville, Alan J. (2009). "Problem-Based Learning and Medical Education Forty Years on". Medical Principles and Practice 18 (1): 1-9. doi: $10.1159 / 000163038$. $\underline{\text { PMID } 19060483}$

[12] Marton F. and Säljö R. (1976) On qualitative differences in learning. I - Outcome and Process' British Journal of Educational Psychology 46, pp. 4-11.

[13] Grimheden, M., Hanson, M., What is Mechatronics? Proposing a Didactical Approach to Mechatronics. Proceedings of the 1st Baltic Sea Workshop on Education in Mechatronics, Kiel, Germany. (2001)

[14] Grimheden, M., Törngren, M., How should Embedded Systems be taught? Experiences and snapshots from Swedish higher engineering education. ACM SIGBED Review 2 (4) (2005), pp. 34-39.

[15] www.incose.org (accessed Aug 2014).

[16] Caspi, P. et al, Guidelines for a graduate curriculum on embedded software and systems, ACM Transactions on Embedded computing systems, Vol 4, No. 3, August 2005, Pages 587-611.

[17] www.eumecha.org (accessed Aug 2014)

[18] Peter Marwedel, Wolfgang Rhode and Katharina Morik, "The TU Dortmund Cyber-Physical Systems Program: A Step Towards Multi-Disciplinary Education." Proceedings of the First Workshop on Cyber-Physical Systems Education (CPS-Ed 2013) at Cyber Physical Systems Week (CPSWeek 2013), Philadelphia, Pennsylvania, USA, April 2013.

Available: http://cpsvo.org/group/edu/workshop/proceedings2013.

[19] http://cps-vo.org/file/7512/download/20240 (accessed Aug. 2014)

[20] Victoria Cengarle, Saddek Bensalem, John McDermid, Roberto Passerone, Alberto Sangiovanni-Vincentelli, Martin Törngren. Characteristics, capabilities, potential applications of Cyber-Physical Systems: a preliminary analysis. Deliverable D2.1 of the CyPhERS FP7 project, Nov. 2013. http://www.cyphers.eu/sites/default/files/D2.1.pdf

[21] Victoria Cengarle, Martin Törngren, Saddek Bensalem, John McDermid, Alberto Sangiovanni-Vincentelli, Roberto Passerone. Structuring of CPS Domain: Characteristics, trends, challenges and opportunities associated with CPS. Deliverable D2.2 of the CyPhERS FP7 project, May 2013. http://www.cyphers.eu/sites/default/files/D2.2.pdf

[22] ICES - Innovative Centre for Embedded Systems. www.ices.kth.se (accessed Aug. 2014).

[23] Martin Törngren, Saddek Bensalem, Victoria Cengarle, DeJiu Chen, John McDermid, Roberto Passerone, Alberto 
Sangiovanni-Vincentelli, Thomas Runkler. CPS: State of the Art. Deliverable D5.1 of the CyPhERS FP7 project, March 2014. http://www.cyphers.eu/sites/default/files/D2.2.pdf

[24] First Workshop on CPS Education, April 8th, 2013, Philadelphia, PA (part of CPSWeek 2013) - accessible at http://cps-vo.org/group/edu/workshop (accessed Aug 2014).

[25] Edward A. Lee and Sanjit A. Seshia, Introduction to Embedded Systems, A Cyber-Physical Systems Approach, http://LeeSeshia.org, ISBN 978-0-557-70857-4, 2011.

[26] "The hunt is on for the Renaissance Man of computing," in The Independent, September 17, 1991.
[27] Hugues, Jérôme and Cardoso, Janette CESEC Chair Training Embedded System Architects for the Critical Systems Domain. (2013) In: 2013 Workshop on Embedded and Cyber-Physical Systems Education, 3 October 2013 (Montreal, Canada).

[28] Mariagiovanna Sami, Miroslaw Malek, Umberto Bondi and Francesco Regazzoni (U Lugano, Switzerland): Embedded Systems Education: Job Market Expectations. Workshop on Embedded and Cyber-Physical Systems Education, at ESWEEK, Oct. 16, 2014, New Delhi, India. 